\title{
Nuclear matter in the chiral limit and the in-medium chiral condensate
}

\author{
O. Plohl and C. Fuchs \\ Institut für Theoretische Physik, Universität Tübingen, \\ Auf der Morgenstelle 14, D-72076 Tübingen, Germany
}

\begin{abstract}
We investigate nuclear matter, i.e. the nuclear equation-of-state (EOS) as well as the relativistic mean fields in the chiral limit. The investigations are based on a chiral nucleon-nucleon EFT interaction where the explicit and implicit pion mass dependence is known up to next-to-leading order. The nuclear bulk properties are found to remain fairly stable in the chiral limit. Based on the same interaction the in-medium scalar condensate is derived, both in Hartree-Fock approximation as well as from the Brueckner G-matrix, making thereby use of the Hellman-Feynman theorem. Short distance physics which determines the reduction of the in-medium nucleon mass is found to play only a minor role for the reduction of the chiral condensate.
\end{abstract}

PACS numbers: 21.30.Fe, 21.65.+f, 24.85.+p

Keywords: Nuclear matter, chiral limit, chiral NN interaction, chiral condensate, effective nucleon mass 


\section{INTRODUCTION}

The investigation of dense nuclear matter is one of the most exciting topics of present days nuclear and hadron physics. Since hadrons are excitations of the QCD vacuum their inmedium excitation spectrum is closely connected to the modification of the QCD vacuum at finite density. The chiral condensate $\langle\bar{q} q\rangle$ is expected to be reduced at finite density and/or temperature which leads to a partial restoration of chiral symmetry of QCD and should be reflected, e.g., in shifts of the corresponding hadron masses. Chiral symmetry is an exact symmetry of QCD in the limit of massless quarks. Since the up and down current quark masses are small, i.e. of the order of 5-10 MeV, this symmetry is still approximately fulfilled. In nature it is, however, spontaneously broken by the non-vanishing - and large expectation value of the scalar quark condensate $\langle\bar{q} q\rangle$ of the QCD vacuum. The spontaneous symmetry breaking, similar to the spontaneous magnetization of a ferromagnet which breaks the symmetry of the underlying Hamiltonian, implies the existence of massless Goldstone bosons which are the pions. The small pion mass of $140 \mathrm{MeV}$ ensures that the concept of chiral symmetry persists as a fundamental feature of low energy hadron physics.

To leading order in density the in-medium scalar condensate drops linearly with the nuclear density with the proportionality given by the pion-nucleon sigma term $\sigma_{N}$. The model independent low density behavior can be derived from the Hellmann-Feynman theorem [1]. To determine the corrections to this leading density dependence a large variety of models has been exploited. These were e.g. the Nambu-Jona-Lasinio (NJL) model [1, 2, 3], various versions of the linear sigma model [4, 5], the Quark Meson Coupling model (QMC) [6] or recently the Polyakov-NJL model [7]. The Hellmann-Feynman theorem relates the inmedium scalar quark condensate with the quark mass derivative of the total energy density. The latter quantity can also be calculated within hadron effective field theory [1, 8 , 9], such as $\sigma \omega$ type models [10] and, more microscopically, within nuclear many-body theory. In a relativistic framework the latter leads to the relativistic Brueckner (DBHF) approach [11, 12, 13] where the nucleon-nucleon interaction is given by boson-exchange models [14]. While

DBHF allows a quite reliable determination of the nuclear equation of state (EOS) up to at least two times nuclear density as a conservative estimate - recent DBHF calculations [15] have been shown to be consistent with astrophysical accelerator based constraints concerning their high density behavior [16] - the unknown quark mass dependence of the mesonic 
couplings and masses introduces large errors when conclusions on the in-medium condensate are drawn [8, 9].

Most hadronic models do, however, not respect chiral symmetry. A more systematic and direct connection to QCD is provided by chiral effective field theory (EFT). Up to now the two-nucleon system has been considered up to next-to-next-to-next-to-leading order $\left(\mathrm{N}^{3} \mathrm{LO}\right)$ in chiral perturbation theory [17, 18, 19]. In such approaches the nucleon-nucleon $(N N)$ potential consists of one-, two- and three-pion exchanges and contact interactions which account for the short-range contributions. The advantage of such approaches is the systematic expansion of the $N N$ interaction in terms of chiral power counting. The expansion is performed in powers of $\left(Q / \Lambda_{\chi}\right)^{\nu}$ where $Q$ is the generic low momentum scale given by the nucleon three-momentum, or the four-momenta of virtual pions or a pion mass. $\Lambda_{\chi} \simeq 4 \pi f_{\pi} \simeq 1$ $\mathrm{GeV}$ is the chiral symmetry breaking scale. Moreover, chiral EFT has a well defined quark mass dependence. For the $N N$ interaction the chiral limit of vanishing current quark and pion masses has been evaluated up to next-to-leading order (NLO) [20, 21] and applied to the deuteron problem [22].

Exploiting the Hellmann-Feynman theorem chiral EFT allows thus a precise determination of the scalar condensate at NLO.

Within finite density QCD sum rules the scalar condensate determines automatically the shift of the effective nucleon mass $M^{*}$. In the sum rule approach scalar and vector fields arise naturally from the structure of the quark propagator which is proportional to the corresponding condensates. The quark correlation function can be expressed to leading order in terms of the scalar condensate $\langle\bar{q} q\rangle_{\rho_{B}}$ and the vector condensate $\left\langle q^{\dagger} q\right\rangle_{\rho_{B}}$ which is introduced by the breaking of Lorentz invariance due to the presence of the medium. The identification of the correlation function with the in-medium nucleon propagator of a dressed quasi-particle leads to scalar and vector self-energies $\Sigma_{\mathrm{s}}$ and $\Sigma_{\mathrm{o}}$ which are of the same order in the condensates [1, 23].

The scalar and vector self-energies $\Sigma_{\mathrm{S}}$ and $\Sigma_{\mathrm{o}}$ are on the other hand key quantities of each relativistic hadronic field theory. They determine the nuclear EOS, the effective nucleon mass $M^{*}=M+\Sigma_{\mathrm{s}}$, the single particle potential $U_{\text {cent }} \simeq \Sigma_{\mathrm{o}}+\Sigma_{\mathrm{s}} \simeq-50 \mathrm{MeV}$ and the spinorbit potential $U_{\text {S.O. }} \propto\left(\Sigma_{\mathrm{o}}-\Sigma_{\mathrm{s}}\right) \simeq 750 \mathrm{MeV}$ in finite nuclei. Relativistic phenomenology implies large fields of opposite sign $\Sigma_{\mathrm{s}} \simeq-350 \mathrm{MeV}$ and $\Sigma_{\mathrm{o}} \simeq+300 \mathrm{MeV}$ 【10, 24]. The same is obtained by relativistic many-body theory, i.e. DBHF [11, 12, 13, 15]. One has, 
however, to keep in mind that the individual scalar and vector components are interpolating fields which do not directly manifest in experimentally accesable observables. Nevertheless, when modern nucleon-nucleon interactions are mapped on a Lorentz covariant operator basis (using projection techniques as briefly described in Section III) they reveal large scalar and vector fields of comparable size in nuclear matter as a model independent fact [25, 26]. This holds not only for manifestly covariantly formulated interactions, such as relativistic One-Boson-Exchange models (Bonn, CD-Bonn, Nijmegen), but also for non-relativistic interactions (Argonne $v_{18}$, Reid93, Idaho $\mathrm{N}^{3} \mathrm{LO}, V_{\text {low k }}$ ) as soon as the symmetries of the Lorentz group are restored. From the analysis of the chiral EFT (Idaho $\mathrm{N}^{3} \mathrm{LO}$ ) potential [17, 18] we found that these fields are generated mainly by contact terms which occur at next-to-leading order in the chiral expansion and which generate the short-range spin-orbit interaction [26].

At moderate nuclear densities the $\mathrm{N}^{3} \mathrm{LO}$ scalar and vector fields were found to be in almost perfect agreement with the prediction from leading order QCD sum rules [26]. The coincidence of the nucleon mass shifts obtained from QCD sum rules and relativistic nuclear phenomenology has been stressed in many works. However, this agreement is not yet fully understood. As pointed out in [27] a naive direct dependence of the nucleon mass $M^{*}$ on the scalar quark condensate leads to contradictions with chiral power counting. Long-distance physics from virtual pions, i.e., the non-analytic term in the expansion of $\sigma_{N}$ gives a sizable contribution to the modification of the in-medium quark condensate. Such contributions are, however, found to play only a minor role for the reduction of the nucleon mass.

In the present work we calculate both quantities, the scalar quark condensate and the effective nucleon mass $M^{*}$ from the same chiral effective interaction. The condensate is determined making use of the Hellmann-Feynman theorem and the fact that, at least up to NLO, the quark mass dependence of the potential is known from its analytic formulation in the chiral limit. The effective mass, on the other hand, can be determined in Hartree-Fock approximation making use of projection techniques on a relativistic operator basis [25, 26]. Since the quark mass dependence is known for the chiral effective interaction up to NLO this allows furthermore to investigate possible changes of the properties of symmetric nuclear matter up to this order. Therefore the large attractive scalar and repulsive vector self-energy components as well as the nuclear equation of state are studied in the chiral limit. This has also been done using projection techniques on a relativistic operator basis in Hartree-Fock 
approximation. Naturally one assumes that the magnitude of these fields persists even in the chiral limit since hadronic properties are not expected to change dramatically in the case of massless quarks or pions. The reason for this is that the expansion of the nuclear force in the context of chiral perturbation theory is well defined for small quark masses and should still be valid in the limit $m_{q} \rightarrow 0$ which is equivalent to $m_{\pi} \rightarrow 0$.

In the first part of the paper a short description of the chiral effective $N N$ interaction is presented. This is followed by the formalism for the relativistic self-energy components in Hartree-Fock approximation. The determination of the relativistic self-energy is based on a projection technique which allows to transform any two-body potential amplitude onto a covariant operator basis or, in other words, to restore the symmetries of the Lorentz group. In the following section the implications for the self-energy components and the equation of state for nuclear matter are shown when going to the chiral limit. In the last section we first present the prediction of the scalar quark condensate in matter derived with the help of the Hellmann-Feynman theorem. This is done within two different approximations, namely Hartree-Fock and the Brueckner ladder approximation. The latter allows to study the influence of short range correlations. Finally the connection between the effective nucleon mass and the scalar quark condensate is discussed.

\section{THE EFT NUCLEON-NUCLEON INTERACTION}

Both, the investigation of the structure of the self-energy in nuclear matter and the resulting EOS in the chiral limit $\left(m_{\pi} \rightarrow 0\right)$ as well as the determination of the scalar condensate in matter depend crucially on the exact knowledge of the implicit and explicit current quark mass dependence of the nuclear force on which the many-body approaches are based on. We are now in the situation to make use of the chiral $N N$ interaction derived in [20] which allows an extrapolation in the pion mass where the quark mass dependence is known analytically up to NLO .

The chiral EFT potential consists of one-, two- and three-pion exchanges and regularizing contact interactions describing the short-range contributions. The chiral expansion of the $N N$ interaction is performed by organizing the contributions in terms of powers of $\left(Q / \Lambda_{\chi}\right)^{\nu}$ where $Q$ is the generic low momentum scale given by the nucleon three-momentum, or the

four-momenta of virtual pions or a pion mass and $\Lambda_{\chi} \simeq 4 \pi f_{\pi} \simeq 1 \mathrm{GeV}$ is the chiral symmetry 
breaking scale.

In [20] the light quark mass dependence of the nuclear force has been derived up to nextto-leading (NLO) order in the framework of a modified Weinberg power counting, i.e., additionally to the one-pion exchange (OPE) potential and contact terms the leading two-pion exchange (TPE) has been considered.

The explicit form of the chiral effective $N N$ potential $V_{\mathrm{NLO}}$ we use is given by [20]

$$
V_{\mathrm{NLO}}=V^{\mathrm{OPE}}+V^{\mathrm{TPE}}+V^{\mathrm{cont}},
$$

where

$$
\begin{aligned}
V^{\mathrm{OPE}}= & -\frac{1}{4} \frac{g_{A}^{2}}{f_{\pi}^{2}}\left(1+2 \Delta-\frac{4 \tilde{m}_{\pi}^{2}}{g_{A}} \bar{d}_{18}\right) \boldsymbol{\tau}_{1} \cdot \boldsymbol{\tau}_{2} \frac{\left(\vec{\sigma}_{1} \cdot \vec{q}\right)\left(\vec{\sigma}_{2} \cdot \vec{q}\right)}{\vec{q}^{2}+\tilde{m}_{\pi}^{2}} \\
V^{\mathrm{TPE}}= & -\frac{\boldsymbol{\tau}_{1} \cdot \boldsymbol{\tau}_{2}}{384 \pi^{2} f_{\pi}^{4}}\left\{L(q)\left[4 \tilde{m}_{\pi}^{2}\left(5 g_{A}^{4}-4 g_{A}^{2}-1\right)+\vec{q}^{2}\left(23 g_{A}^{4}-10 g_{A}^{2}-1\right)+\frac{48 g_{A}^{4} \tilde{m}_{\pi}^{4}}{4 \tilde{m}_{\pi}^{2}+\vec{q}^{2}}\right]\right. \\
& \left.\quad+\vec{q}^{2} \ln \frac{\tilde{m}_{\pi}}{m_{\pi}}\left(23 g_{A}^{4}-10 g_{A}^{2}-1\right)\right\} \\
& -\frac{3 g_{A}^{4}}{64 \pi^{2} f_{\pi}^{4}}\left(L(q)+\ln \frac{\tilde{m}_{\pi}}{m_{\pi}}\right)\left\{\vec{\sigma}_{1} \cdot \vec{q} \vec{\sigma}_{2} \cdot \vec{q}-\vec{q}^{2} \vec{\sigma}_{1} \cdot \vec{\sigma}_{2}\right\} \\
V^{\mathrm{cont}}= & \bar{C}_{S}+\bar{C}_{T}\left(\vec{\sigma}_{1} \cdot \vec{\sigma}_{2}\right)+\tilde{m}_{\pi}^{2}\left(\bar{D}_{S}-\frac{3 g_{A}^{2}}{32 \pi^{2} f_{\pi}^{4}}\left(8 f_{\pi}^{2} C_{T}-5 g_{A}^{2}+2\right) \ln \frac{\tilde{m}_{\pi}}{m_{\pi}}\right) \\
& +\tilde{m}_{\pi}^{2}\left(\bar{D}_{T}-\frac{3 g_{A}^{2}}{64 \pi^{2} f_{\pi}^{4}}\left(16 f_{\pi}^{2} C_{T}-5 g_{A}^{2}+2\right) \ln \frac{\tilde{m}_{\pi}}{m_{\pi}}\right)\left(\vec{\sigma}_{1} \cdot \vec{\sigma}_{2}\right) \\
& +C_{1} \vec{q}^{2}+C_{2} \vec{k}^{2}+\left(C_{3} \vec{q}^{2}+C_{4} \vec{k}^{2}\right)\left(\vec{\sigma}_{1} \cdot \vec{\sigma}_{2}\right) \\
& +i C_{5} \frac{\vec{\sigma}_{1}+\vec{\sigma}_{2}}{2} \cdot(\vec{k} \times \vec{q})+C_{6}\left(\vec{q} \cdot \vec{\sigma}_{1}\right)\left(\vec{q} \cdot \vec{\sigma}_{2}\right)+C_{7}\left(\vec{k} \cdot \vec{\sigma}_{1}\right)\left(\vec{k} \cdot \vec{\sigma}_{2}\right)
\end{aligned}
$$

with $g_{A}$ and $f_{\pi}$ the physical values of the nucleon axial coupling and the pion decay constant, respectively. Because at NLO any shift in $g_{A}$ and $f_{\pi}$ for a different value of $m_{\pi}$ in the TPE is a $\mathrm{N}^{4} \mathrm{LO}$ effect for the TPE the physical values $g_{A}=1.26$ and $f_{\pi}=92.4 \mathrm{MeV}$ are used. The value of the pion mass is indicated by $\tilde{m}_{\pi}$ compared to the physical one denoted by $m_{\pi}$. $L(q)$ is given by

$$
L(q) \equiv L(|\vec{q}|)=\frac{\sqrt{4 \tilde{m}_{\pi}^{2}+\vec{q}^{2}}}{|\vec{q}|} \ln \frac{\sqrt{4 \tilde{m}_{\pi}^{2}+\vec{q}^{2}}+|\vec{q}|}{2 \tilde{m}_{\pi}} .
$$

$\Delta$ represents the relative shift in the ratio $g_{A} / f_{\pi}$ compared to its physical value since they show an implicit dependence on the pion mass

$$
\Delta \equiv \frac{\left(g_{A} / f_{\pi}\right)_{\tilde{m}_{\pi}}-\left(g_{A} / f_{\pi}\right)_{m_{\pi}}}{\left(g_{A} / f_{\pi}\right)_{m_{\pi}}}
$$




$$
=\left(\frac{g_{A}^{2}}{16 \pi^{2} f_{\pi}^{2}}-\frac{4}{g_{A}} \bar{d}_{16}+\frac{1}{16 \pi^{2} f_{\pi}^{2}} \bar{l}_{4}\right)\left(m_{\pi}^{2}-\tilde{m}_{\pi}^{2}\right)-\frac{g_{A}^{2} \tilde{m}_{\pi}^{2}}{4 \pi^{2} f_{\pi}^{2}} \ln \frac{\tilde{m}_{\pi}}{m_{\pi}} .
$$

The low-energy constants (LECs) $\bar{C}_{S, T}$ and $\bar{D}_{S, T}$ appear at LO and are related to the $C_{S, T}$ from [19] via

$$
C_{S, T}=\bar{C}_{S, T}+m_{\pi}^{2} \bar{D}_{S, T}
$$

The LECs $\bar{D}_{S, T}$ have not been fixed by experiment till now. In Ref. [20] natural values have been assumed for these constants

$$
\bar{D}_{S, T}=\frac{\alpha_{S, T}}{f_{\pi}^{2} \Lambda_{\chi}^{2}}, \quad \text { where } \quad \alpha_{S, T} \sim 1 \quad \text { and } \quad \Lambda_{\chi} \simeq 1 \mathrm{GeV}
$$

The LECs $\bar{d}_{16}, \bar{d}_{18}$ and $\bar{l}_{4}$ are related to pion-nucleon interactions. We take $\bar{l}_{4}=4.3$ which is fixed with relatively small error bars. The LECs $\bar{d}_{16}, \bar{d}_{18}$ are not yet uniquely fixed, i.e. there exists a certain range of possible values fixed from different observables. The implications on the results induced by the uncertainties of the LECs will be discussed later in greater detail.

\section{COVARIANT REPRESENTATION AND THE NUCLEON SELF-ENERGY}

In the following the mean field in nuclear matter is determined by calculating the relativistic self-energy $\Sigma$ in Hartree-Fock approximation at tree level. It provides therefore a qualitative rather than a quantitative description of the nuclear many-body problem. Nevertheless the scale of these fields is already set at tree level. Although essential for nuclear binding and saturation, higher order correlations, in particular short-range correlations, change the size of the fields by less than 25\%, as has been estimated in [26] comparing HF to a full selfconsistent relativistic DBHF calculation. However, for nuclear binding these deviations are essential. To meet the empirical saturation point of nuclear matter one has to introduce a self-consistent scheme and to account for short-range correlations. Such calculations have to be based on the in-medium T-matrix (or G-matrix) rather than the bare potential $V$. G-matrix correlations will be discussed in connection with the chiral condensate in the next section.

Here we shortly sketch the formalism for the evaluation of the self-energy. More details can be found in Refs. [26] and [13]. 
The self-energy for a nucleon with four-momentum $k$ follows from the interaction $V$ by integrating over the occupied states $q$ in the Fermi sea

$$
\Sigma_{\alpha \beta}\left(k, k_{F}\right)=-i \int \frac{d^{4} q}{(2 \pi)^{4}} G_{\tau \sigma}^{D}(q) V^{A}(k, q)_{\alpha \sigma ; \beta \tau} .
$$

The evaluation of the Hartree integral is sufficient because the matrix elements are fully antisymmetrized containing direct (Hartree) and exchange (Fock) contributions. To determine the self-energy only positive-energy states are taken into account as done in the standard DBHF approach. The Dirac propagator

$$
G^{D}(q)=[\not q+M] 2 \pi i \delta\left(q^{2}-M^{2}\right) \Theta\left(q_{0}\right) \Theta\left(k_{F}-|\mathbf{q}|\right)
$$

describes the on-shell propagation of a nucleon with momentum $\mathbf{q}$ and energy $E_{\mathbf{q}}=$ $\sqrt{\mathbf{q}^{2}+M^{2}}$ inside the Fermi sea. Due to the $\Theta$ functions in the propagator only positive energy nucleons are allowed in the intermediate scattering states which prevents the occurence of divergent contributions coming from negative energy states.

Based on symmetry considerations in isospin saturated symmetric nuclear matter the selfenergy can be written as a sum of a scalar $\Sigma_{\mathrm{s}}$, a time-like vector $\Sigma_{\mathrm{o}}$ and a spatial vector part $\Sigma_{\mathrm{v}}$. Thus, in nuclear matter rest frame the Dirac structure of the self-energy has the simple form

$$
\Sigma\left(k, k_{\mathrm{F}}\right)=\Sigma_{\mathrm{s}}\left(k, k_{\mathrm{F}}\right)-\gamma_{0} \Sigma_{\mathrm{o}}\left(k, k_{\mathrm{F}}\right)+\gamma \cdot \mathbf{k} \Sigma_{\mathrm{v}}\left(k, k_{\mathrm{F}}\right)
$$

The Lorentz components of the self-energy operator (9) are then given by [13]

$$
\begin{aligned}
\Sigma_{\mathrm{S}} & =\frac{1}{4} \int^{k_{F}} \frac{d^{3} \mathbf{q}}{(2 \pi)^{3}} \frac{M}{E_{\mathbf{q}}}\left[4 g_{\mathrm{S}}-g_{\tilde{\mathrm{S}}}+4 g_{\mathrm{A}}-\frac{\left(k^{\mu}-q^{\mu}\right)^{2}}{4 M^{2}} g_{\widetilde{\mathrm{PV}}}\right], \\
\Sigma_{\mathrm{o}} & =\frac{1}{4} \int^{k_{F}} \frac{d^{3} \mathbf{q}}{(2 \pi)^{3}}\left[g_{\tilde{\mathrm{S}}}-2 g_{\mathrm{A}}+\frac{E_{\mathbf{k}}}{E_{\mathbf{q}}} \frac{\left(k^{\mu}-q^{\mu}\right)^{2}}{4 M^{2}} g_{\widetilde{\mathrm{PV}}}\right] \\
\Sigma_{\mathrm{v}} & =\frac{1}{4} \int^{k_{F}} \frac{d^{3} \mathbf{q}}{(2 \pi)^{3}} \frac{\mathbf{k} \cdot \mathbf{q}}{|\mathbf{k}|^{2} E_{\mathbf{q}}}\left[g_{\tilde{\mathrm{S}}}-2 g_{\mathrm{A}}+\frac{k_{z}}{q_{z}} \frac{\left(k^{\mu}-q^{\mu}\right)^{2}}{4 M^{2}} g_{\widetilde{\mathrm{PV}}}\right]
\end{aligned}
$$

To evaluate the self-energy operator in the nuclear matter rest frame the two-body interaction matrix $V$ determined in the two-particle centre-of-mass (c.m.) frame and usually given in the $|J L S\rangle$ basis, has to be represented covariantly by Dirac operators and Lorentz invariant amplitudes [28], a procedure which can be applied to any two-body amplitude. This is also the most convenient way to Lorentz-transform the interaction matrix from one frame into another [29]. 
Naturally a fully relativistic treatment invokes the excitation of anti-nucleons. However, standard $N N$ potentials (even OBE type potentials such Bonn, CD-Bonn or Nijmegen) are restricted to the positive energy sector and neglect the explicit coupling to anti-nucleons. As a consequence one has to work in a subspace of the full Dirac space. This shortcoming can be avoided using fully covariant potentials which explicitely include anti-nucleon states [30, 31]. The present investigations and those in Refs. [25, 26] have, however, been restricted to "standard" potentials based on the no sea approximation. Similarly, the EFT potentials [17, 18] applied here and in our previous investigations do not explicitly include anti-nucleons, in contrast to covariant approaches which require renormalization procedures to restore chiral power counting [32].

Working in the positive energy subspace, symmetry arguments and the restriction to on-shell scattering allow the two-body matrix elements to be are expanded in terms of five Lorentz invariants. A possible choice of a set of five linearly independent operators are the scalar, vector, tensor, axial-vector and pseudo-scalar Fermi covariants $\Gamma_{m}=\{\mathrm{S}, \mathrm{V}, \mathrm{T}, \mathrm{P}, \mathrm{A}\}$ with

$$
\mathrm{S}=1 \otimes 1, \quad \mathrm{~V}=\gamma^{\mu} \otimes \gamma_{\mu}, \quad \mathrm{T}=\sigma^{\mu \nu} \otimes \sigma_{\mu \nu}, \quad \mathrm{P}=\gamma_{5} \otimes \gamma_{5}, \quad \mathrm{~A}=\gamma_{5} \gamma^{\mu} \otimes \gamma_{5} \gamma_{\mu}
$$

The choice of the operator basis is not unique. In [13] it has been shown that the so-called complete $p v$ representation is an appropriate choice where the set of covariants originally proposed by Tjon and Wallace [28] is given by

$$
\Gamma_{m}=\{\mathrm{S},-\tilde{\mathrm{S}},(\mathrm{A}-\tilde{\mathrm{A}}), \mathrm{PV},-\widetilde{\mathrm{PV}}\}
$$

PV and $\widetilde{\mathrm{PV}}$ are the direct and exchange pseudo-vector covariants, analogous to the pseudoscalar covariant $\mathrm{P}$, however, with $\gamma_{5}$ replaced by $\left(\not q^{\prime}-\not q\right) / 2 M \gamma_{5}$. A PV vertex suppresses the coupling to negative states and it is consistent with soft pion theorems based on chiral symmetry considerations.

Thus the on-shell $\left(|\mathbf{q}|=\left|\mathbf{q}^{\prime}\right|\right)$ scattering matrix is given by

$$
\hat{V}^{I}\left(\mathbf{q}^{\prime}, \mathbf{q}\right)=\sum_{m} g_{m}^{I}(|\mathbf{q}|, \theta) \Gamma_{m}
$$

where $\theta$ is the c.m. scattering angle and $I=0,1$ the isospin channel. For the Hartree-Fock self-energy it is sufficient to consider $\theta=0$ when anti-symmetrized matrix elements are used since $\theta=\pi$ contains then only redundant information. The transformation of the Born amplitudes from an angular-momentum basis onto the covariant basis (15) is now standard 
and runs over the following steps: $|L S J\rangle \rightarrow$ partial wave helicity states $\rightarrow$ plane wave helicity states $\rightarrow$ covariant basis. The first two transformation can e.g. be found in Refs. [33]. The last step has to be performed numerically by matrix inversion [13, 29].

The expressions for the NLO chiral potential given in Eqs. (22)-(44) are independent of the nucleon mass. Nevertheless the nucleon mass appears in the expressions for the calculation of the relativistic mean fields, Eq. (12), as well as in the procedure of projecting the two-body amplitudes on the covariant basis. Moreover, the quark mass dependence of the nucleon mass is also the leading term which determines the quark mass dependence of the EOS. The EOS, i.e. the energy per particle $\mathrm{E} / \mathrm{A}$ is given by

$$
\mathrm{E} / \mathrm{A}=\frac{1}{\rho_{B}} \int_{F} \frac{d^{3} \mathbf{k}}{2 \pi^{3}}\left[\frac{k^{2}}{2 M}+\frac{1}{2} U_{\text {s.p. }}\left(k, k_{\mathrm{F}}\right)\right]
$$

with the single particle potential $U_{\text {s.p. }}\left(k, k_{\mathrm{F}}\right)$ defined through the fields

$$
U_{\text {s.p. }}\left(k, k_{\mathrm{F}}\right)=\frac{M}{E} \Sigma_{\mathrm{s}}-\frac{k_{\mu} \Sigma^{\mu}}{E}=\frac{M \Sigma_{\mathrm{s}}}{\sqrt{\mathbf{k}^{2}+M^{2}}}-\Sigma_{\mathrm{o}}+\frac{\Sigma_{\mathrm{v}} \mathbf{k}^{2}}{\sqrt{\mathbf{k}^{2}+M^{2}}} .
$$

The integration in Eq. (16) runs over the Fermi sea $F$ and we account for the full momentum dependence of the self-energy components $\Sigma_{\mathrm{s}}, \Sigma_{\mathrm{o}}, \Sigma_{\mathrm{v}}$.

Within the framework of chiral EFT the physical (vacuum) nucleon mass $M$ can be expressed as

$$
M=M_{0}+\sigma_{N}
$$

where $M_{0}$ is the value of the nucleon mass in the chiral limit. The sigma term $\sigma_{N}$ represents the contribution from explicit chiral symmetry breaking to the nucleon mass and determines the quark mass dependence of the nucleon mass

$$
\sigma_{N}=\sum_{q=u, d} m_{q} \frac{d M}{d m_{q}}=m_{\pi}^{2} \frac{d M}{d m_{\pi}^{2}}
$$

which, through $m_{\pi}^{2} \sim m_{q}$ translates into a dependence on the pion mass. The chiral limit of the nucleon mass and of the sigma term, respectively, has been evaluated up to NNLO [34], where the corrections to the NLO dependence were, however, found to be small.

In order to be consistent with the $N N$ interaction we account in the following for the pion mass dependence of the nucleon mass at NLO (expressions given in [34]) when the self-energy components and the EOS are studied in the chiral limit. 

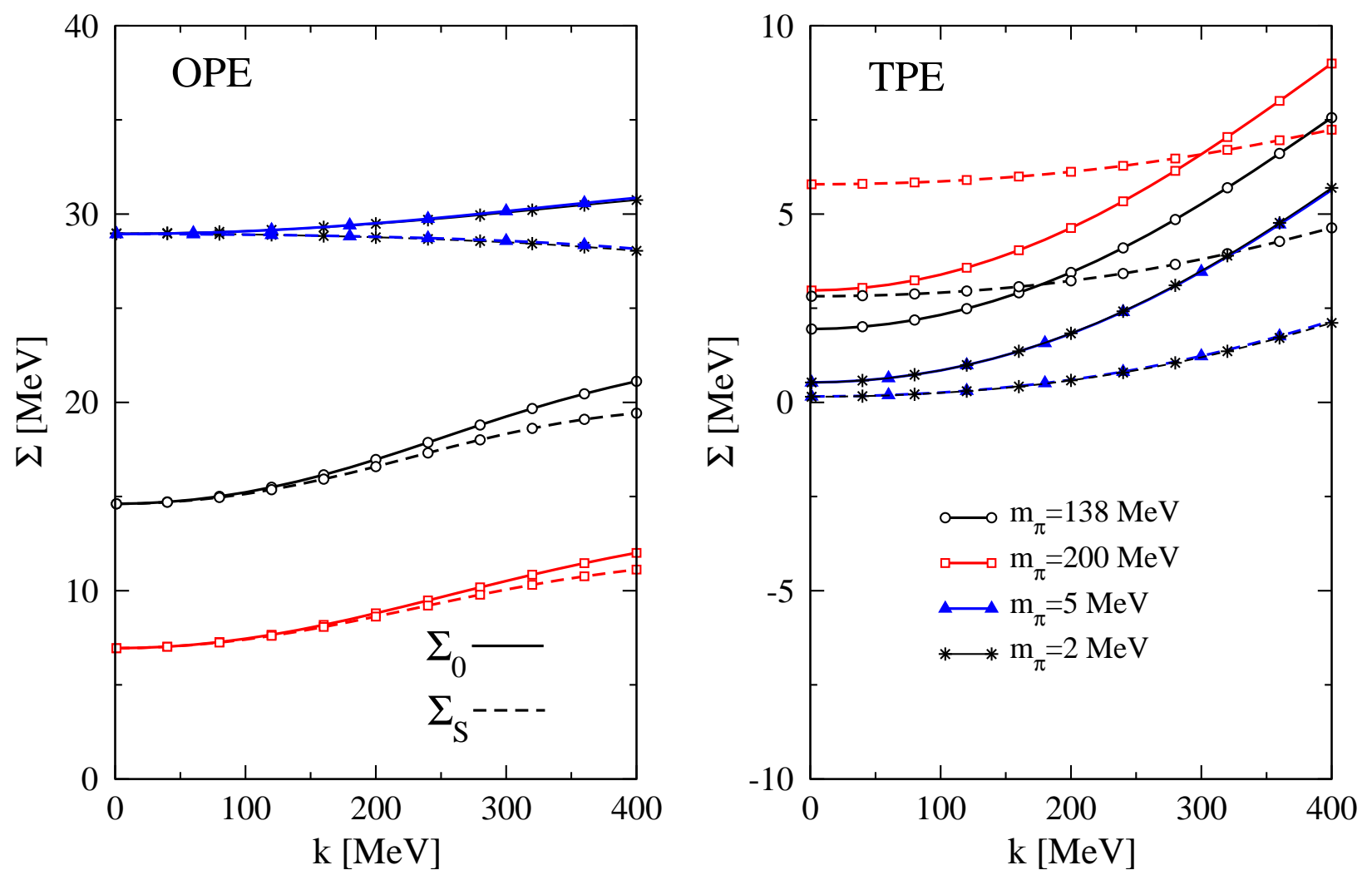

FIG. 1: (Color online) The tree level scalar (dashed lines) and vector (full lines) self-energy components in matter at $k_{\mathrm{F}}=1.35 \mathrm{fm}^{-1}$ are shown for different values of the pion mass $m_{\pi}$. The NLO one-pion exchange (left panel) and two-pion exchange (right panel) are shown. The one-pion exchange is obtained with $\bar{d}_{16}=-1.23$ and $\bar{d}_{18}=-0.97$.

\section{NUCLEAR MATTER IN THE CHIRAL LIMIT}

The analysis of the EFT potential performed in [26] revealed that the scalar/vector fields are generated by contact terms which occur at NLO order in the chiral expansion. These are four-nucleon contact terms with two derivatives which generate the short-range spinorbit interaction. The strength of the corresponding low energy constants, in particular those connected to the spin-orbit force, is dictated by $P$-wave $N N$ scattering data. Pion dynamics as well as LO and $\mathrm{N}^{3} \mathrm{LO}$ contacts provide only corrections to the fields generated by the NLO contact terms. Thus one could expect that the quark mass dependence of the fields is mainly determined by the quark mass dependence of the contact terms which is moderate. However, before coming to the full self-energy, we consider the contributions from one-pion (OPE) and two-pion-exchange (TPE) separately. Fig. 1 shows the scalar 
$\Sigma_{\mathrm{s}}$ and vector $\Sigma_{\mathrm{o}}$ self-energy components from the next-to-leading order OPE and TPE contributions at a Fermi momentum of $k_{\mathrm{F}}=1.35 \mathrm{fm}^{-1}$ which corresponds to a nuclear density of $\rho_{B}=0.166 \mathrm{fm}^{-3}$. As a well known result, at the physical pion mass the scalar and vector self-energy components from the pseudo-vector OPE are of the same sign and of moderate strength. This is also true in the chiral limit. The self-energy components $\Sigma_{\mathrm{s}}, \Sigma_{\mathrm{o}}$ approach a constant value of about $30 \mathrm{MeV}$. For the not yet uniquely fixed LECs entering into the expression for the renormalized OPE the values $\bar{d}_{16}=-1.23$ and $\bar{d}_{18}=-0.97$ have been taken [20]. The uncertainty due to these LECs $\bar{d}_{16,18}$ does not significantly affect the scalar and vector fields. The same is true for the corresponding EOS (see below). This is, however, not the case what concerns the scalar quark condensate as discussed in detail in the following chapter.

The scalar $\Sigma_{\mathrm{s}}$ and vector $\Sigma_{\mathrm{o}}$ self-energy components generated by the TPE are already small for the physical case $\left(m_{\pi}=138 \mathrm{MeV}\right)$ and are further reduced by $\approx 2,5 \mathrm{MeV}$ in the chiral limit. At zero momentum both components almost vanish and show a slight increase with increasing momentum. As for the OPE the fields are repulsive and approach a constant value in the chiral limit.

Next we will consider the role of the contact terms. The contact terms connected to the LECs $C_{1 \ldots 7}$, Eq. (4), do not depend on the pion mass at NLO. Since the magnitude of the scalar and vector self-energy components is mainly set by contact interactions connected to the spin-orbit force where the strength is proportional to the LEC $C_{5}$ in Eq. (4) the modification of the fields in the chiral limit can in total be expected to be moderate. The pion mass dependent part of the contact interactions, i.e., the first to lines in Eq. (44) provides only small contributions.

The uncertainties due to the not yet uniquely fixed LECs $\bar{d}_{16,18}$ entering the renormalized OPE (2) do not strongly affect the self-energy components. However, a second source of uncertainty appears in the the part of the contact interactions connected to the not known LECs $\bar{D}_{S, T}$ which depend on the pion mass. In Ref. [20] this range of uncertainty has been explored through an independent variation of the parameters $\alpha_{S, T}$ in Eq. (8) in the range of $-3.0<\alpha_{S, T}<3.0$. In Ref. [20] this rather wide variation of the LECs $\bar{D}_{S, T}$ was motivated by a wide range of possible parameter sets of NLO LECs fitted with different cut-off combinations. However, in the present case - using the Idaho chiral potential - we are restricted to one parameter set with a general cut-off of $\lambda=500 \mathrm{MeV}$. A variation of the 

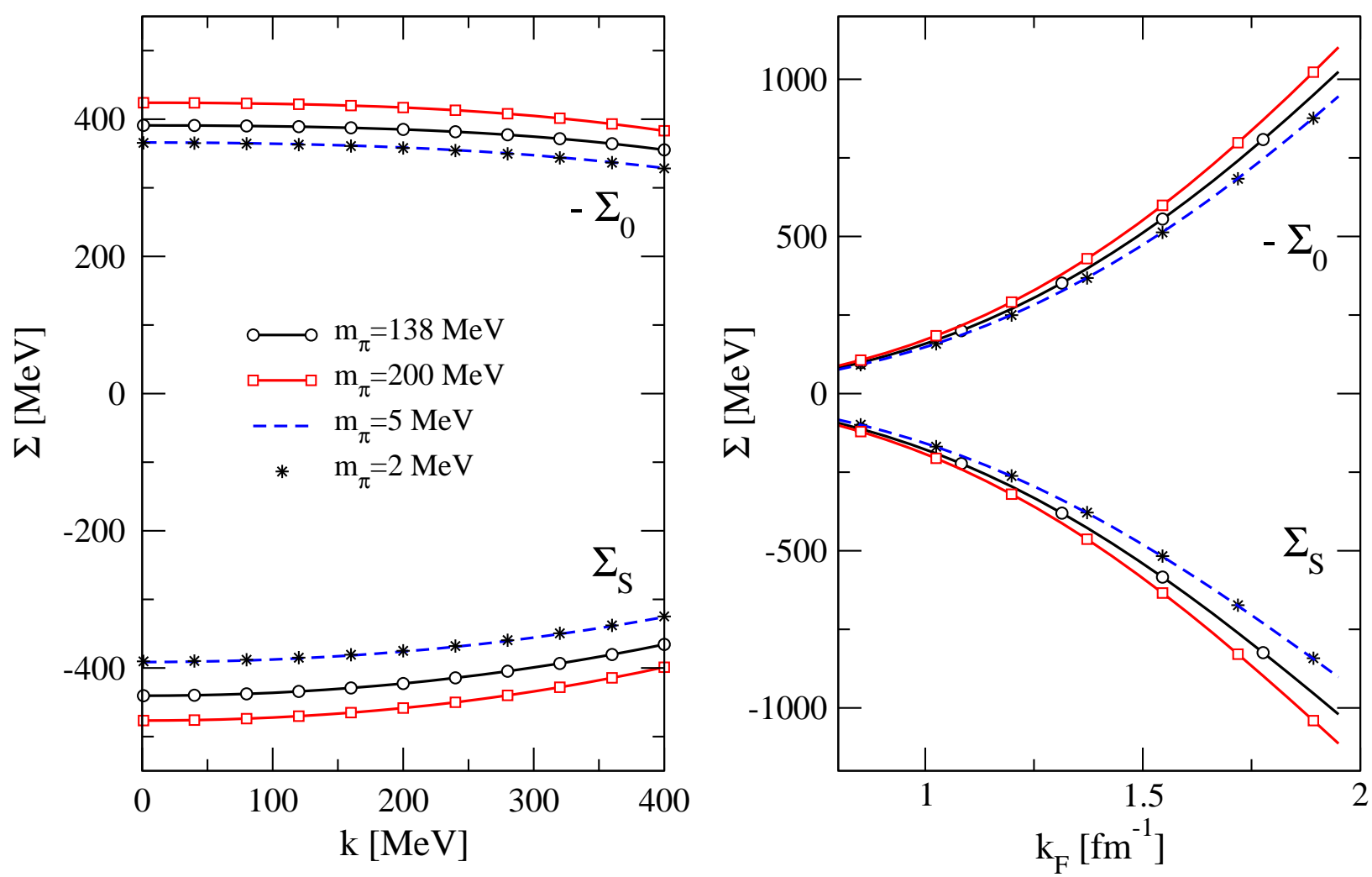

FIG. 2: (Color online) Left: momentum dependence of the tree level scalar and vector self-energy components in nuclear matter at $k_{\mathrm{F}}=1.35 \mathrm{fm}^{-1}$ evaluated for different values of the pion mass $m_{\pi}$. Right: tree level scalar and vector self-energy components in nuclear matter as a function of the Fermi momentum $k_{\mathrm{F}}$ for different values of the pion mass $m_{\pi}$.

LECs over a wide range is therefore likely to overestimate the uncertainty originating from the LECs $\bar{D}_{S, T}$. Therefore we restrict the present discussion to values $\alpha_{S, T} \approx 1$. Results turned out to be stable against a variation of $\alpha_{S}$ and $\alpha_{T}$ in the same direction, i.e. small deviations from combinations of $\alpha_{S, T}$ where both parameters are close to each other do practically not change the results.

In Fig. 2 the full tree-level self-energy components are now shown as a function of the momentum $k$. An approximately vanishing pion mass, i.e. $m_{\pi}=2 \mathrm{MeV}$ and $m_{\pi}=5 \mathrm{MeV}$, leads to a small reduction of the repulsive vector field $(\approx 30 \mathrm{MeV})$ and of the attractive scalar field $(\approx 50 \mathrm{MeV})$, respectively. The same can be seen on the right hand side in Fig. 2 where the fields are shown as a function of the Fermi momentum $k_{\mathrm{F}}$. An increase of the pion mass to $m_{\pi}=200 \mathrm{MeV}$ leads to an opposite behavior.

In summary, a careful analysis of the chiral EFT $N N$ interaction leads to large scalar and 
vector fields which essentially maintain their strength in the chiral limit, however, with the tendency of a slight decrease of absolute size.

In this context it may be interesting to compare this behavior with the naive assumption of a dropping $\sigma$ meson mass within the framework of Quantum-Hadron-Dynamics (QHD) [10]. In this case the scalar and vector fields are inverse proportional to the masses of the $\sigma$ and $\omega$ mesons

$$
\Sigma_{\mathrm{s}}=-\frac{g_{\sigma}^{2}}{m_{\sigma}^{2}} \rho_{S} \quad, \quad \Sigma_{\mathrm{o}}=+\frac{g_{\omega}^{2}}{m_{\omega}^{2}} \rho_{B}
$$

where $g_{\sigma}$ and $g_{\omega}$ are the corresponding meson-nucleon coupling constants and $\rho_{S} \sim \rho_{B}$ is the scalar nucleon density. The assumption of dropping $\sigma$ and $\omega$ meson masses according to a naive Brown-Rho scaling [35] together with fairly constant couplings would lead to a strong increase of scalar and vector fields in size. Chiral EFT predicts the opposite behavior, namely slightly decreasing fields. Interpreting this result in terms of the simple QHD picture means that the ratio of coupling functions and meson masses in Eq. (201) has to stay fairly constant. Assuming dropping meson masses the coupling functions should show the same density dependence. Such a scenario is not completely unrealistic since in the framework of density dependent relativistic mean field theory [36] where density dependent meson coupling functions $g_{\sigma, \omega}^{2}\left(\rho_{B}\right)$ are derived from the Brueckner G-matrix [37, 38] or fitted to finite nuclei [39, 40], such a behavior is usually obtained.

It is clear that a rather small reduction of the scalar and vector fields has only moderate consequences for the EOS in the chiral limit. How the change in the fields affects the nuclear EOS is depicted in Fig. 3. However, before coming to the pion mass dependence we shortly discuss the tree level EOS derived from the chiral EFT potential. The EOSs for isospin symmetric nuclear matter at the various orders of the potential are shown on the left hand side of Fig. 3. There appear large jumps in the EOS when going from LO up to $\mathrm{N}^{3} \mathrm{LO}$. As discussed in [26] the contact terms which generate the large attractive/repulsive scalar/vector potentials arise at NLO. Contributions from higher order provide corrections to these potentials. These are moderate on the scale of the fields of several hundred MeV magnitude. However, due to the subtle cancellation between scalar attraction and vector repulsion such corrections may be large on the scale of the binding energy, i.e. several tenth of $\mathrm{MeV}$. This behavior is exactly reflected in the EOSs shown in Fig. 3 which jump from unbound at LO to over-bound at NLO and $\mathrm{N}^{2} \mathrm{LO}$ to loosely bound at $\mathrm{N}^{3} \mathrm{LO}$. 

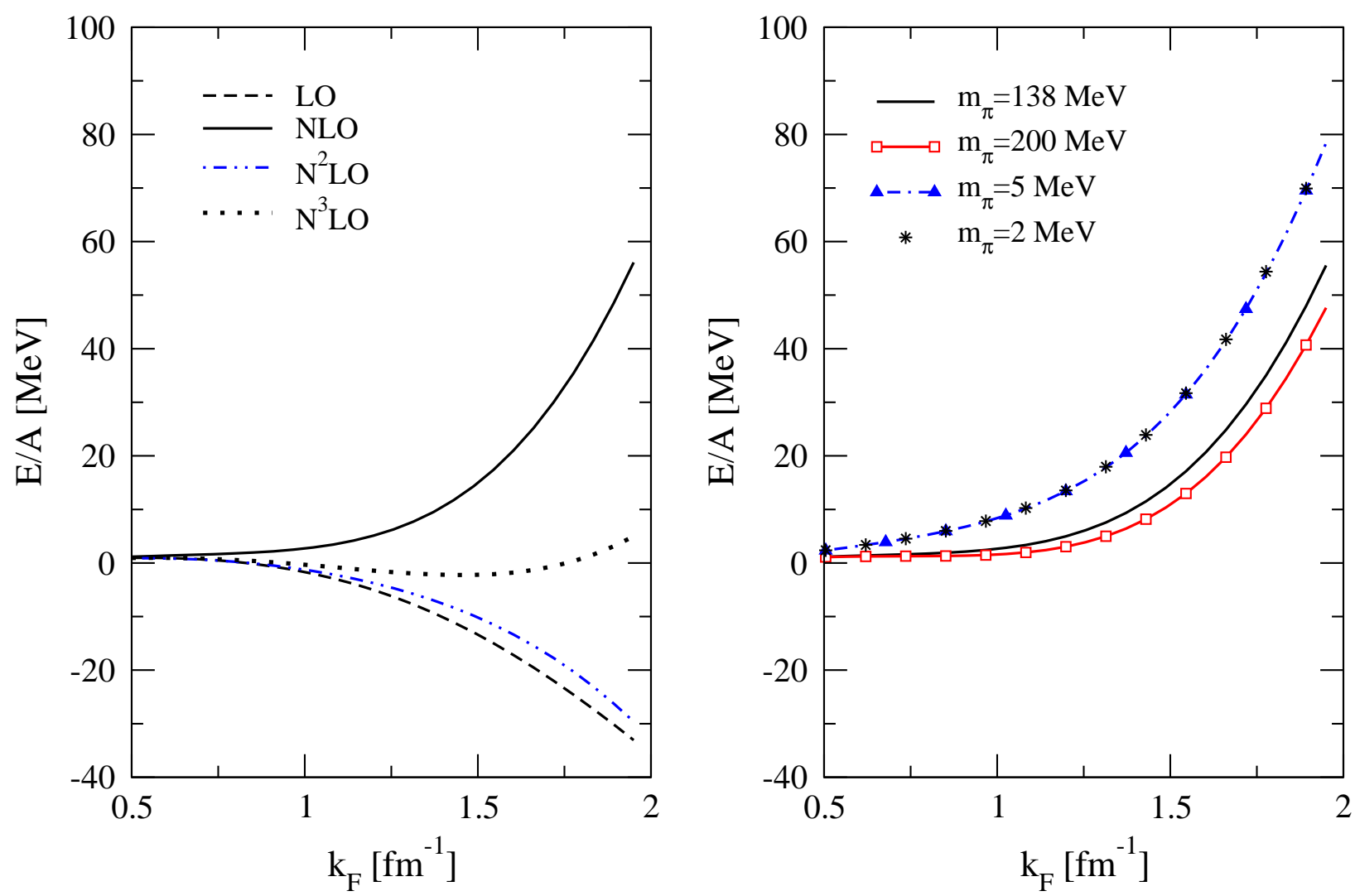

FIG. 3: (Color online) Hartree-Fock calculation of the nuclear equation of state, i.e. the energy per particle $E / A$ as a function of the Fermi momentum $k_{F}$. On the left hand side the tree level results are shown order by order up to $\mathrm{N}^{3} \mathrm{LO}$. On the right hand side the pion mass dependence of the EOS at NLO is shown again, i.e. the result for the physical case of $m_{\pi}=138 \mathrm{MeV}$ is compared to the case of $m_{\pi}=2 \mathrm{MeV}, m_{\pi}=5 \mathrm{MeV}$ and $m_{\pi}=200 \mathrm{MeV}$.

The present tree level calculation is of course not a realistic microscopic nuclear matter calculation which would on the one hand require to apply the $\mathrm{N}^{3} \mathrm{LO}$ force, and secondly, to perform a self-consistent summation of the Brueckner ladder diagrams [13, 15]. The difference between a tree-level and a full relativistic Brueckner calculation has been discussed in [26]. However, the NLO tree level calculation allows a consistent investigation of the chiral limit at the order at which the pion mass dependence of the chiral $N N$ potential has been derived.

This is done in the left panel of Fig. 3 which compares the NLO EOS at the physical pion mass $m_{\pi}=138 \mathrm{MeV}$ to that at $m_{\pi}=200 \mathrm{MeV}, m_{\pi}=2 \mathrm{MeV}$ and $m_{\pi}=5 \mathrm{MeV}$, respectively. Doing so, one observes a slight softening of the repulsive NLO EOS when the pion mass is 
increased from its physical value to $m_{\pi}=200 \mathrm{MeV}$. In the chiral limit the scalar attraction is slightly stronger reduced than the vector repulsion (see Fig. 2). The EOS becomes therefore more repulsive. One can expect that the observed effect survives also when a full Brueckner ladder is summed. Since the pion mass dependence of the contact terms is rather weak, Brueckner short range correlations can not be expected to change the results dramatically. As already pointed out, the main source of uncertainty arises from the unknown LECs $\bar{D}_{S, T}$ entering the renormalized contact forces. Concerning the tree level EOS, we did, however, not find any tendency of a qualitative change even for large, probably unrealistic variations of the dimensionless coefficients $\alpha_{S, T}$. In a full Brueckner calculation the attraction may be increased since iterated OPE and TPE is known to be quenched by Pauli blocking of the intermediate states in the Bethe-Goldstone, respectively the Bethe-Salpeter equation.

Therefore the conclusion is, that the magnitude of the large scalar and vector fields in matter persists in the chiral limit and that the physics of infinite nuclear matter is similar to that of a vanishing pion mass. This finding is in agreement with the investigations made in [41]. In this work nuclear matter was analyzed taking basically the chiral limit of the OPE and assuming that the short range and the intermediate range part of any $N N$ potential is not affected.

\section{IN-MEDIUM SCALAR CONDENSATE}

The spontaneous breakdown of chiral symmetry involves a qualitative rearrangement of the QCD ground state, due to the appearance of scalar quark-antiquark pairs. The corresponding non-vanishing ground-state expectation value $\langle\bar{q} q\rangle$, denoted as the scalar quark condensate, is an order parameter of spontaneous chiral symmetry breaking. Any reduction of the scalar density of quarks in matter can therefore be interpreted as a sign of partial restoration of chiral symmetry.

\section{A. Determination from Hellmann-Feynman theorem}

The vacuum value of the lowest-dimensional quark condensate is about [42]

$$
\langle\bar{q} q\rangle_{0} \simeq-(225 \pm 25 \mathrm{MeV})^{3}
$$


The density dependence of the chiral condensate $\langle\bar{q} q\rangle$ can be extracted exploiting the Hellmann-Feynman theorem with respect to the symmetry breaking current quark mass term of the QCD Hamiltonian. We consider isospin symmetric matter making thereby use of the isospin symmetry of the condensates $(\langle\bar{q} q\rangle \equiv\langle\bar{u} u\rangle \simeq\langle\bar{d} d\rangle)$. Defining $\bar{q} q \equiv \frac{1}{2}(\bar{u} u+\bar{d} d)$ and $m_{q} \equiv \frac{1}{2}\left(m_{u}+m_{d}\right)$ the quark mass term is given by $2 m_{q} \bar{q} q$. Isospin-breaking terms are neglected. With the help of the Hellmann-Feynman theorem one obtains the in-medium quark condensate by determining the energy density $\mathcal{E}$ of nuclear matter

$$
2 m_{q}\left(\langle\bar{q} q\rangle_{\rho_{B}}-\langle\bar{q} q\rangle_{0}\right)=m_{q} \frac{d \mathcal{E}}{d m_{q}} .
$$

The derivative is taken at fixed density. The energy density of nuclear matter is given by

$$
\mathcal{E}=M \rho_{B}+\frac{E\left(\rho_{B}\right)}{A} \rho_{B}
$$

where the second term of $\mathcal{E}$ is the energy per particle $E / A$ (times the baryon density), i.e. the contributions from the nucleon kinetic energy and nucleon-nucleon interactions. Inserting the energy density $\mathcal{E}$ into Eq. (22) and using the Gell-Mann, Oakes, Renner (GOR) relation

$$
2 m_{q}\langle\bar{q} q\rangle_{0}=-m_{\pi}^{2} f_{\pi}^{2}
$$

with the definition of the pion-nucleon $\sigma_{N}$ term from Eq. (19)

$$
\frac{d M}{d m_{q}}=\frac{\sigma_{N}}{m_{q}}
$$

one obtains

$$
\frac{\langle\bar{q} q\rangle_{\rho_{B}}}{\langle\bar{q} q\rangle_{0}}=1-\frac{\rho_{B}}{m_{\pi}^{2} f_{\pi}^{2}}\left[\sigma_{N}+m_{q} \frac{d}{d m_{q}} \frac{E}{A}\right] .
$$

The derivative of the energy per particle with respect to the quark mass can be re-expressed using the chain rule

$$
\frac{\langle\bar{q} q\rangle_{\rho_{B}}}{\langle\bar{q} q\rangle_{0}}=1-\frac{\rho_{B}}{m_{\pi}^{2} f_{\pi}^{2}}\left[\sigma_{N}+m_{q} \frac{\partial(E / A)}{\partial M} \frac{d M}{d m_{q}}+m_{q} \frac{\partial(E / A)}{\partial m_{\pi}} \frac{d m_{\pi}}{d m_{q}}\right] .
$$

The derivative of the pion mass using Eq. (24) is given by

$$
\frac{d m_{\pi}}{d m_{q}}=\frac{m_{\pi}}{2 m_{q}}
$$

valid to leading order in chiral perturbation theory. Introducing

$$
\rho^{\chi}=\frac{m_{\pi}^{2} f_{\pi}^{2}}{\sigma_{N}}
$$


one finally obtains

$$
\frac{\langle\bar{q} q\rangle_{\rho_{B}}}{\langle\bar{q} q\rangle_{0}}=1-\frac{\rho_{B}}{\rho^{\chi}}\left[1+\frac{\partial(E / A)}{\partial M}+\frac{\partial(E / A)}{\partial m_{\pi}} \frac{m_{\pi}}{2 \sigma_{N}}\right] .
$$

The first term on the r.h.s. of Eq. (30) which reduces the condensate in matter is model independent and of first order in the nuclear density [1, 23]. Inserting the empirical value of $\sigma_{N}=(45 \pm 7) \mathrm{MeV}$ for the sigma term [43] and taking $m_{\pi}=138 \mathrm{MeV}$ and $f_{\pi}=92.4$ $\mathrm{MeV}$ one finds in Table $\mathbb{I}$, that the in-medium scalar condensate is to leading order in density approximately $\frac{1}{3}$ smaller than its vacuum value at nuclear saturation density. In the following a value of $\rho_{0}=0.173 \mathrm{fm}^{-3}$, corresponding to a Fermi momentum of $k_{F}=1.37$ $\mathrm{fm}^{-1}$, is chosen as the standard value for the nuclear saturation density.

From Fig. 4 one sees that to leading order a complete restoration of chiral symmetry would already occur at $\rho_{B} \approx 2.7 \rho_{0}$. Such a scenario is unrealistic and contradictory to the knowledge from heavy ion reactions [44] and astrophysics, e.g. neutron stars [16]. Hence, one has to account for higher order corrections in density coming from the $d(E / A) / d m_{q}$ term in Eq. (26). One might estimate this correction to be small due to the binding energy of $E / A \approx-16 \mathrm{MeV}$, which is almost two orders of magnitude smaller than the nucleon mass contributing dominantly to the energy density in Eq. (23). Nevertheless, since the quark mass derivative of the interaction energy is the relevant quantity, it is by far not obvious that higher order corrections are negligible.

Thus, a reliable extraction of the density dependent scalar condensate $\langle\bar{q} q\rangle_{\rho_{B}}$ requires both, a sophisticated nuclear matter calculation and the exact knowledge of the current quark mass dependence of all model parameters entering into the energy density.

Previous estimates of the scalar condensate based on sophisticated ab-initio many-body approaches [8, 9] suffered from this problem. The relativistic Brueckner approach chosen in [8, 9] provides a reliable description of nuclear matter bulk properties. Such calculations are based on realistic $N N$ potentials, e.g. one-boson exchange potentials [14]. However, the current quark mass dependences of the model parameters, i.e. meson masses and coupling constants, are unknown to large extent and have therefore either been roughly estimated or even been neglected [8, 9].

In [8] it was found that the largest uncertainty in the calculation of the in-medium quark condensate $\langle\bar{q} q\rangle_{\rho_{B}}$ arises due to the unknown quark mass dependence of the scalar isoscalar $\sigma$ meson exchange which parameterizes effectively correlated two-pion exchange. 
In the present work the determination of the energy per particle $E / A$ will be determined within Hartree-Fock (HF), Eq. (16), and in a second step within the Brueckner-HartreeFock (BHF) approximation. Since the $N N$ interaction is thereby based on chiral EFT, Eqs. (22)-(44), where the complete pion mass dependence is known up to NLO [20] we are free of uncertainties concerning unknown quark mass derivatives. Remaining ambiguities when applying the Hellman-Feynman theorem are only due to the not yet uniquely fixed LECs $\bar{D}_{S, T}$ in the NLO contact terms, see Eq. (4), and the LECs $\bar{d}_{16,18}$ showing up in the OPE exchange. The uncertainties coming from these LECs will be discussed.

In Hartree-Fock approximation nuclear matter is normally unbound, in particular when high precision OBE type potentials are applied. The situation turns out to be qualitatively different for low momentum interactions like $V_{\text {low } \mathrm{k}}$ and Idaho $\mathrm{N}^{3} \mathrm{LO}$ (see Fig. 3) where the hard core is strongly suppressed by high momentum cut-offs. In this case isospin saturated nuclear matter collapses and Brueckner ladder correlations do not improve on [45, 46]. In this case the repulsion generated by three-body forces which appear at $\mathrm{N}^{2} \mathrm{LO}$ turned out to be essential to stabilize nuclear matter and to obtain reasonable saturation properties [46]. The inclusion of three-body forces is beyond the scope of the present work, in particular what concerns the pion mass dependence, but for a quantitative determination of the EFT EOS they should be included.

However, for a reliable estimate of the in-medium condensate the role of $N N$ correlations, in particular short-range and tensor correlations, has to be considerd. One might assume that $N N$ correlations influence the result for the condensate, in particular at higher densities. In order to estimate their importance the self-consistent iteration scheme of BHF theory is applied.

The central equation of the BHF approximation is the Bethe-Goldstone equation

$$
\mathcal{G}(\omega)=V+V \frac{Q}{\omega-H_{0}} \mathcal{G}(\omega)
$$

$V$ is the bare interaction and $Q$ the Pauli operator which prevents from scattering into occupied intermediate states below the Fermi momentum $k_{F}$. The starting energy is denoted by $\omega$. The operator $H_{0}$ defines the energy spectrum of the intermediate two-particle state where we use the so called continuous choice. Therefore the single-particle energies for particles as well as for hole states above the Fermi surface are calculated from the kinetic energy and a mean field part which has to be determined from the $\mathcal{G}$ matrix self-consistently. 
The single-particle energies are then given by

$$
\epsilon_{\alpha}^{B H F}=\epsilon_{\alpha}+\sum_{\mu \leq F}\left\langle\alpha \mu\left|\mathcal{G}\left(\omega=\epsilon_{\alpha}^{B H F}+\epsilon_{\mu}^{B H F}\right)\right| \alpha \mu\right\rangle
$$

These single-particle energies are then parameterized in terms of an effective mass and a constant potential, i.e. $\epsilon_{k} \approx k^{2} / 2 M^{*}+U$.

In the present work we apply the non-relativistic approach since it is not possible to use the chiral $N N$ potential in a relativistic BHF calculation (DHBF) [13, 15] where one accounts in addition for the dressing of the potential matrix elements $V \mapsto V^{*}$. The latter requires, however, a definite relativistic structure of the interaction, like for covariant OBE-type potentials. Nevertheless, differences between a relativistic and a non-relativistic treatment should be moderate concerning the derivative of the EOS with respect to the current quark mass.

The prediction of the in-medium scalar condensate in both approaches, i.e., HF and BHF are shown in Fig. 4. As expected, deviations from the leading order result due to $N N$ interactions and nucleon kinetic energy, Eq. (30), increase with density. For both approaches, $\mathrm{HF}$ and $\mathrm{BHF}$, the additional contributions lead to a weaker reduction of the in-medium quark condensate. Especially in the case of the BHF calculation the leading order prediction provides a very good description of the quark condensate up to a density of $0.8 \rho_{B}$.

At nuclear saturation density $\rho_{0}$ the reduction of the in-medium quark condensate is about $3 \%(\mathrm{BHF})$ and 12\% (HF) smaller compared to leading order. Deviations are, however, growing with density, where at $\rho_{B} \approx 2 \div 3 \rho_{0}$ the quark condensate is reduced to $\approx 35 \%$ $(\mathrm{HF})$ and $\approx 30 \%(\mathrm{BHF})$. Naturally the BHF approach is more reliable in this density region. However, in summary effects from short-range $N N$ correlations and the quenching of OPE and TPE due to Pauli blocking, both present in BHF, have only minor implications for the condensate as can be seen from the comparison to the HF result.

The uncertainty due to the not yet uniquely fixed LECs $\bar{d}_{16,18}$ in the renormalized OPE, Eq. (2) which was already mentioned in the context of the EOS in the chiral limit, enters also into the determination of the scalar condensate. However, now this uncertainty is much more severe. The light shaded bands in Fig. 4 indicate the range of possible variations: The LEC $\bar{d}_{18}$ is extracted from the Goldberger-Treiman discrepancy. We take the three empirically found values given in [20] extracted from three different phase shift, $\bar{d}_{18}=-0.84$ $\mathrm{GeV}^{-2}$ [47], $\bar{d}_{18}=-0.97 \mathrm{GeV}^{-2}$ [48] and $\bar{d}_{18}=-1.54 \mathrm{GeV}^{-2}$ [49], respectively. Furthermore 

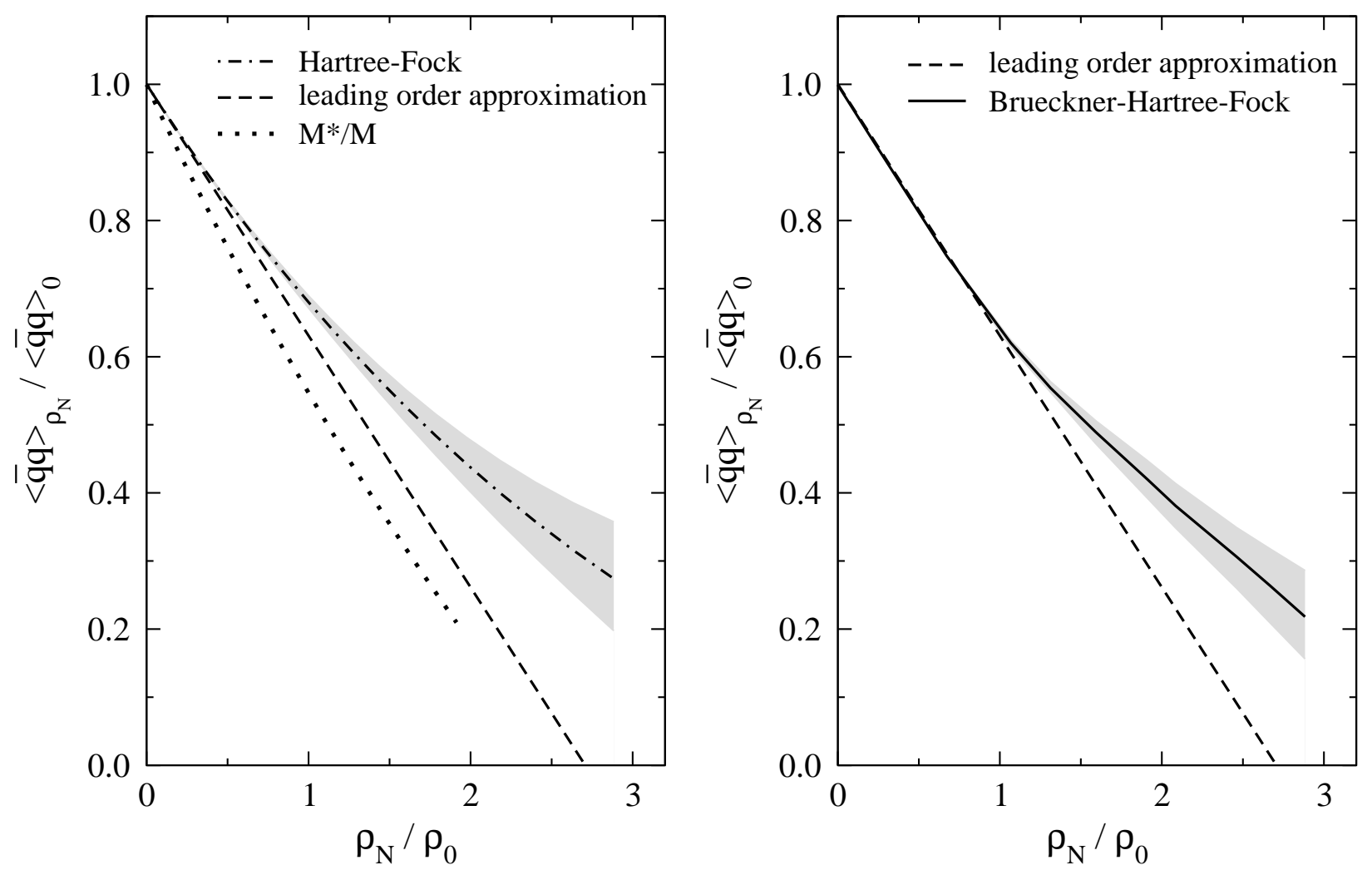

FIG. 4: In-medium scalar quark condensate as a function of density with $\sigma_{N}=45 \mathrm{MeV}$ obtained in various approximations: A Hartree-Fock calculation of $\langle\bar{q} q\rangle_{\rho_{B}} /\langle\bar{q} q\rangle_{0}$ is shown compared to $M^{*} / M$ where $M^{*}=M+\Sigma_{\mathrm{s}}$ is evaluated at tree level in Hartree-Fock approximation (left). A full calculation based on the Brueckner-Hartree-Fock approach is shown on the right. The light shaded bands indicate the uncertainty of the corresponding result varying the LECs $\bar{d}_{16}$ from $\bar{d}_{16}=-0.91$ $\mathrm{GeV}^{-2}$ to $\bar{d}_{16}=-1.76 \mathrm{GeV}^{-2}$ and $\bar{d}_{18}$ from $\bar{d}_{18}=-0.84 \mathrm{GeV}^{-2}$ to $\bar{d}_{18}=-1.54 \mathrm{GeV}^{-2}$. Dashed line: model-independent leading order result.

we vary the LEC $\bar{d}_{16}$ in the range from $\bar{d}_{16}=-0.91$ to $\bar{d}_{16}=-1.76$ as done in [20]. The upper bound of the shaded band corresponds to $\bar{d}_{16}=-1.76$ and $\bar{d}_{18}=-0.84$ whereas the lower bound corresponds to $\bar{d}_{16}=-0.91$ and $\bar{d}_{18}=-1.54$. These uncertainties are also given in Table 【. The HF (dash-dotted line) and BHF (solid line) mean values are obtained with $\bar{d}_{16}=-1.23$ and $\bar{d}_{18}=-0.97$.

Comparing with previous approaches performed in a similar spirit [8, 9] we find generally a stronger reduction of the scalar condensate. In [9] calculations were based on the relativistic DBHF approach and an one-boson-exchange potential (Bonn A) has been used. In this approach an unexpected increase of the in-medium scalar condensate at densities above 
TABLE I: Predictions of $\langle\bar{q} q\rangle_{\rho_{B}} /\langle\bar{q} q\rangle_{0}$ obtained with the Hellmann-Feynman theorem in diverse approximations compared with $M^{*} / M$ for three different values of the nucleon density $\rho_{B}$.

\begin{tabular}{ccccc}
\hline \hline & \multicolumn{2}{c}{ Hellmann-Feynman theorem } & M* $/ \mathbf{M}$ \\
$\rho_{B} / \rho_{0}$ & Leading order & HF & BHF & in HF \\
\hline 0.5 & 0.815 & $0.828 \pm 0.002$ & $0.812 \pm 0.001$ & 0.759 \\
1.0 & 0.630 & $0.677 \pm 0.010$ & $0.641 \pm 0.004$ & 0.546 \\
1.5 & 0.445 & $0.550 \pm 0.020$ & $0.510 \pm 0.014$ & 0.354 \\
\hline \hline
\end{tabular}

$\rho<2.5 \rho_{B}$ has been found. The same tendency, i.e. an increasing quark condensate at high density has been observed in [8]. In [9] it was assumed that this increase is caused by a breakdown of the underlying assumptions related to the current quark mass dependences of the model parameters, i.e. meson masses and coupling constants. The authors concluded that the use of not chirally invariant $N N$ potentials may lead to wrong predictions in a density region where chiral restoration is expected to occur.

As already mentioned we do not face such problems since the chirally invariant EFT interaction used in the present work has a well defined quark mass dependence. The only source of uncertainty arise due to the LECs $\bar{d}_{16,18}$ which are not yet uniquely fixed and the unknown LECs $\bar{D}_{S, T}$ entering the short-range part, i.e., the contact force which could provide substantial corrections to the scalar quark condensate. Nevertheless, the same argument given in the previous section kept us from showing a wide undefined variation. Therefore, we restrict our calculation again to the case of $\alpha_{S, T} \approx 1$. However, as for the EOS, the prediction of the quark condensate is not considerably altered varying $\alpha_{S, T}$ for combinations of $\alpha_{S, T}$ where both parameters are close to each other. The contributions which change the condensate originate then mainly from TPE and renormalized contact forces. The LECs $C_{1 \ldots 7}$ in Eq. (41) do not depend on the pion mass after renormalization and the related contact terms do therefore not contribute to the change of the in-medium quark condensate.

Nevertheless, considering the possible band of variation due to the LECs $\bar{d}_{16,18}$, both, the HF and BHF calculations shown in Fig. 4 do not indicate saturation or even an increase of the condensate in the considered density range up to $3 \rho_{B}$. Extrapolating the BHF prediction to high densities a complete restoration of chiral symmetry, i.e., a vanishing scalar quark 
condensate is not likely to happen below $4 \rho_{0}$, even if one takes the range of uncertainty from not yet exactly known LECs into account.

The first determination of the in-medium quark condensate adopting the Hellmann-Feynman theorem has been carried out by Cohen et al. [1], based on the $\pi-N$ Fock term. There the condensate was found to be reduced to a value of 0.694 at nuclear saturation density $\rho_{0}$ and 0.58 at $1.5 \rho_{0}$ which is in fair agreement with $0.677 \pm 0.01$ and $0.550 \pm 0.020$ obtained in the present HF calculation (third column of Table I). This agreement, is, however, somewhat accidental since we find that TPE and contact interactions (which both have not been included in [1]) reduce the contribution from OPE by $\approx 50 \%$. Moreover, in [1] the $d g_{\pi N} / d m_{q}$ dependence has been neglected and a different value for $g_{\pi N}$ has been used. Both calculations are, however, comparable in the sense that $N N$ correlations are neglected and they are of the same order in the density. Moreover, short-range physics due to contact terms, which have been neglected in [1] are found to provide only moderate corrections as can be seen from Fig. 5 ,

In Fig. 5 the derivatives $\partial(E / A) / \partial m_{\pi}$ from Eq. (30) as a function of the density are shown with respect to the different contributions coming from pion dynamics, Eqs. (2) and (3), and from the contact terms ( $V^{c}$ on, Eq. (4) , respectively. The dashed-dotted line indicates the derivative $\partial(E / A) / \partial M$. The contribution coming from OPE is negative and considerably larger compared to those from TPE and contact interactions. In general the contributions from pion dynamics, i.e., OPE and TPE are smaller in BHF due to quenching effects. In the case of the contact interactions (44), one has to keep in mind that the short-range terms $\tilde{m}_{\pi}^{2} \ln \tilde{m}_{\pi}$ show up due to TPE and the renormalization of the leading order contact terms by pion loops.

The contribution from nucleon interactions is getting substantially more important with increasing density compared to the contribution from the nucleon kinetic energy (dot-dashed line) which is of order $O\left(\rho^{5 / 3}\right)$. Thus the nuclear interaction provides important corrections to the Fermi gas approximation usually made in QCD sum rule approaches.

It turns out that the calculations are highly sensitive to the pion mass dependence of the pion nucleon coupling constant $g_{\pi N}$ which has been often neglected in earlier works. This fact can also be seen from the relatively large bands of uncertainty in Fig. 4 since the corresponding LECs enter into the relative shift of $g_{A} / F_{\pi}$, Eq. ([6]), which is connected to $g_{\pi N}$ via the Goldberger-Treiman relation $g_{\pi N} / M=g_{A} / F_{\pi}$. 

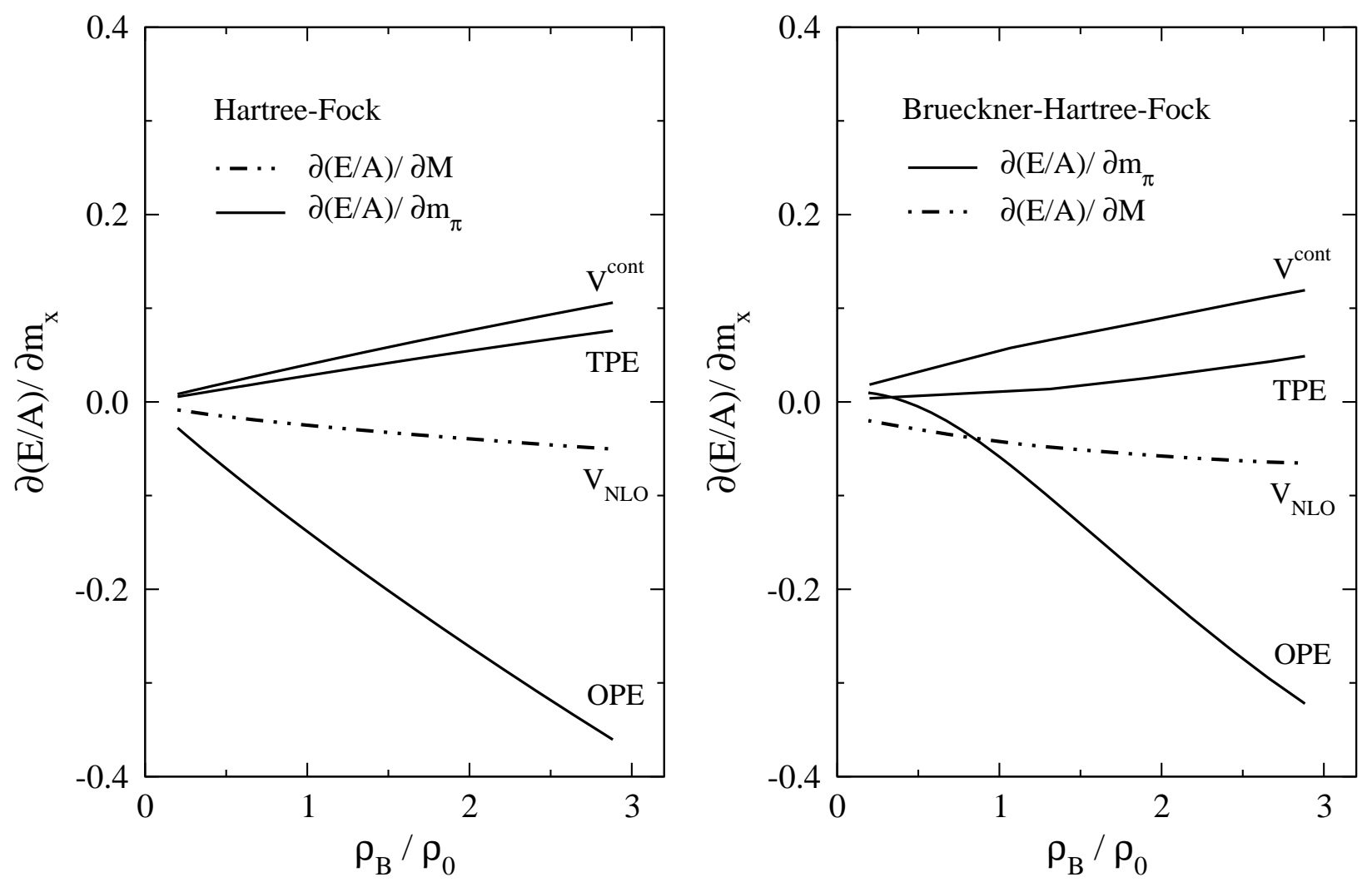

FIG. 5: The derivatives $\partial(E / A) / \partial m_{\pi}$ as a function of density with respect to the full NLO calculation as well as the separate contributions, i.e. OPE, TPE and contact interactions are shown. Additionally the dash-dotted line denotes $\partial(E / A) / \partial M$ for the full NLO calculation.

We conclude that the contributions from nucleon interactions to the change of the scalar condensate in matter are mainly due to low-momentum virtual pions. In contrast to the scalar/vector fields which are generated by NLO contact interactions [25] the contact terms and short-range correlations, i.e., the short-distance physics, seem to play a minor role for the change of the in-medium quark condensate. Nevertheless, for a fully reliable prediction of the in-medium quark condensate the little known LECs $\bar{D}_{S, T}$ entering the NLO contact interactions, Eq. (41), have to be fixed with better precision.

\section{B. Effective nucleon mass}

QCD in-medium sum rules relate the scalar and vector in-medium condensates $\langle\bar{q} q\rangle_{\rho_{B}}$ and $\left\langle q^{\dagger} q\right\rangle_{\rho_{B}}$ to the isoscalar scalar and vector self-energies of a nucleon in matter. Thus the model independent leading order result which should be valid at low density determines the density 
dependence of the effective nucleon mass $M^{*}=M+\Sigma_{\mathrm{s}}$ within the in-medium QCD sum rule approach. The scalar and vector fields $\Sigma_{\mathrm{s}}$ and $\Sigma_{\mathrm{o}}$ arise naturally from the structure of the quark propagator which is proportional to the corresponding in-medium quark condensate. The quark correlation function which follows from the operator product expansion can be written to leading order in terms of scalar $\langle\bar{q} q\rangle_{\rho_{B}}$ and vector condensates $\left\langle q^{\dagger} q\right\rangle_{\rho_{B}}$. In contrast to the scalar condensate to leading order the vector condensate is exactly known. It is given by the quark density in the nuclear matter rest-frame since the baryon current is conserved, i.e. $\left\langle q^{\dagger} q\right\rangle_{\rho_{N}}=3 / 2 \rho_{B}$. In [26] we compared the NLO EFT vector self-energy to the leading order sum rule result. As in the case of the scalar self-energy deviations were found to be small at moderate densities. The next order in the operator product expansion involves four-quark operators and combinations of quark and gluon fields which are often neglected due to their highly non-trivial structure. Attempts to fix the density dependence of higher order contributions in the operator product expansion have e.g. been performed in [50]. Here we restrict the present discussion to the scalar field $\Sigma_{\mathrm{s}}$ which follows automatically identifying the correlation function with the in-medium nucleon propagator of a dressed quasi-particle [1, 23]

$$
\Sigma_{\mathrm{s}}=-\frac{8 \pi^{2}}{\Lambda_{B}^{2}}\left[\langle\bar{q} q\rangle_{\rho_{B}}-\langle\bar{q} q\rangle_{0}\right]=-\frac{8 \pi^{2}}{\Lambda_{B}^{2}} \frac{\sigma_{N}}{m_{u}+m_{d}} \rho_{S} .
$$

The expression on the right is usually obtained using GOR (24) and the model independent term for the scalar condensate in matter which depends linearly on the nucleon density $\rho_{B}$ in Eq. (26). Consequently the expression is of leading order in density where $\rho_{S}$ is the scalar nucleon density. The Borel mass scale $\Lambda_{B} \simeq 4 \pi f_{\pi} \simeq 1 \mathrm{GeV}$ is the generic low energy scale of $\mathrm{QCD}$ which separates the non-perturbative from the perturbative regime. It coincides with the chiral symmetry breaking scale $\Lambda_{\chi}$ of ChPT. Applying Ioffe's formula [51] for the nucleon mass $M \simeq-\frac{8 \pi^{2}}{\Lambda_{B}^{2}}\langle\bar{q} q\rangle$ and the GOR relation one finally obtains [52]

$$
\Sigma_{\mathrm{s}}(\rho)=-\frac{\sigma_{N} M}{m_{\pi}^{2} f_{\pi}^{2}} \rho_{B}
$$

where the difference between the scalar and vector density, $\rho_{S}$ and $\rho_{B}$, can be neglected at low densities $k_{F}^{2} \ll M^{2}$. For the ratio $M^{*} / M$ follows then

$$
\frac{M^{*}}{M}=\frac{M+\Sigma_{\mathrm{s}}}{M}=\frac{\sigma_{N}}{m_{\pi}^{2} f_{\pi}^{2}} \rho_{B} .
$$

Naturally, Eq. (35) represents the model independent leading order prediction for $\langle\bar{q} q\rangle_{\rho_{B}} /\langle\bar{q} q\rangle_{0}$, i.e., the leading order term in Eq. (26). Equalizing the two quantities, i.e., 
scalar condensate and effective nucleon mass, is, however, not as straightforward as Eq. (35) would suggest [26, 27]. As already stated in Ref. [27] a direct dependence of the nucleon mass $M^{*}$ on the in-medium condensate contradicts chiral power counting. Moreover, the inmedium quark condensate contains contributions from low-momentum virtual pions, which do not contribute to the properties of the nucleon in matter.

With the present formalism at hand, we are able to perform a consistent comparison of the in-medium scalar condensate, derived from the Hellmann-Feynman theorem, and the effective nucleon mass where both are obtained from the same chiral EFT interaction and at the same order.

In Fig. 4 also the ratio $M^{*} / M$ is shown with the effective nucleon mass given by $M^{*}=M+\Sigma_{\mathrm{s}}$. The scalar field $\Sigma_{\mathrm{s}}$ is determined from the chiral EFT potential at NLO in HF approximation, making use of projection techniques on a relativistic operator basis as described in Section III. As one can see also from Table I, at saturation density the effective mass $M^{*}$ or the ratio $M^{*} / M$, respectively, is reduced to a value of about 0.546 and is decreasing approximately linear up to $2 \rho_{0}$. The reduction of the effective mass $M^{*}$ is about $\approx 13 \%$ larger at $\rho_{0}$ than that of the scalar condensate $(\mathrm{HF})$, see also Table $\mathbb{I}$. At $1.5 \rho_{0}$ the difference is about $20 \pm 2 \%$. Thus the approximation of Eq. (34) does not hold. By a naive comparison of the two quantities the in-medium condensate may contribute at the utmost by about $\approx 80 \%$ to the change of the the nucleon mass in matter at $1.5 \rho_{0}$.

As already mentioned, the higher order contributions in Eq. (30) from the nucleon interaction are mainly due to OPE and TPE, i.e., low-momentum virtual pions give the main contribution to the change of the scalar quark condensate. The appearance of the large scalar field $\Sigma_{\mathrm{s}}$ which enters the effective nucleon mass $M^{*}=M+\Sigma_{\mathrm{s}}$ originates on the other hand from NLO contact interactions (to be more precise from the part which is connected to the spin-orbit force), i.e., it is driven by short distance physics [26]. Low-momentum pion dynamics is negligible concerning the appearance of the large scalar and vector fields $\Sigma_{\mathrm{s}}$ and $\Sigma_{\mathrm{o}}$ at the considered order (NLO). The present investigations confirm thus the considerations of Ref. [27] which were based on a chiral expansion of the sigma term.

In summary, a direct dependence of the properties of the nucleon mass on the in-medium scalar quark condensate as suggested by Eq. (35) can be ruled out. 


\section{SUMMARY}

We investigated nuclear bulk properties in the chiral limit $m_{\pi} \rightarrow 0$. This concerns both, the EOS as well as scalar and vector self-energy fields in matter. The large relativistic selfenergy components are obtained by restoring the symmetries of the Lorentz group of the nuclear interaction. The essential ingredient on which the present investigations are based is the chiral EFT nuclear force where the implicit and explicit pion mass dependence is known analytically up to NLO and allows a well defined extrapolation in the pion mass [20].

We found that nuclear bulk properties remain fairly stable in the chiral limit. This is true for the EOS as well as the magnitude of the scalar and vector mean fields. Both quantities are mainly affected by the pion mass dependence of the OPE and TPE. In such investigations a source of uncertainty remains due to the not completely constrained LECs $\bar{D}_{S, T}$ which appear in the renormalized contact forces. Nevertheless a qualitative change concerning the properties of the EOS in the chiral limit is not likely to happen.

Furthermore we have calculated the density dependence of the chiral order parameter or scalar quark condensate in nuclear matter making use of the Hellmann-Feynman theorem which relates the scalar quark condensate with the current quark mass derivative of the nuclear energy density. As above, the energy density was calculated in Hartree-Fock approximation. However, to be more realistic and to include also short range correlations we applied also the Brueckner-Hartree-Fock approximation. Since the quark mass dependence of the chiral $N N$ interaction is known up to NLO this approach is free from any ambiguities which arise concerning the analytic and chiral structure of the potential. The quark mass dependence of the pion-nucleon coupling constant $g_{\pi N}$ has thereby been taken into account and was found to be important. Uncertainties due to unknown low-energy constants entering the pion-nucleon coupling constant $g_{\pi N}$ do not change the results qualitatively.

Higher order corrections from the nucleon kinetic and interaction energy become significantly more important above saturation density when compared to the model independent leading order prediction for the scalar quark condensate. They lead in general to a weaker reduction of the in-medium quark condensate and do not indicate a complete restoration of chiral symmetry in the density range where hadronic models are reliable. Since Hartree-Fock and Brueckner-Hartree-Fock provide quantitatively comparable results one can conclude that short-range correlations and quenching effects, both present in Brueckner theory, have only 
minor implications for the density dependence of the quark condensate. The substantial contributions from nucleon interactions are due to low-momentum virtual pions, i.e., OPE and TPE. Short-distance physics in terms of contact terms and short-range correlations have no important impact on the in-medium properties of the quark condensate.

This present formalism allows also to perform a consistent comparison of the in-medium scalar condensate, derived directly from the Hellmann-Feynman theorem, and the effective nucleon mass $M^{*}=M+\Sigma_{\mathrm{s}}$ where the scalar self-energy $\Sigma_{\mathrm{s}}$ enters. For the first time both quantities were derived from the same chiral EFT interaction and at the same order. In general the effective nucleon mass calculated in the many-body approach is smaller $(\approx 10 \%$ at $\rho_{0}$ ) then the model independent leading order prediction which is used in the QCD sum rule approach.

Moreover, in the present investigations it turns out, that the reduction of the two quantities, namely the in-medium condensate and the in-medium nucleon mass, are of different physical origin. While the latter is dominantly generated by short distance physics in terms of NLO contact interactions [26] virtual low-momentum pions provide the essential contributions responsible for the change of the in-medium scalar quark condensate.

\section{Acknowledgments}

We thank R. Machleidt for providing the Idaho N3LO program package and H. Muether for helpful conversation and moreover providing his Brueckner-Hartree-Fock code. This work was supported by the DFG under contract GRK683 (European Graduate School TübingenBasel-Graz). 
[1] T.D. Cohen, R.J. Furnstahl, D.K. Griegel, Phys. Rev. Lett. 67 (1991) 961; Phys. Rev. C 45 (1992) 1881.

[2] S. Klimt, M. Lutz, W. Weise, Phys. Lett. B 249 (1990) 386.

[3] T. Maruyama, K. Tsushima and A. Faessler, Nucl. Phys. A 535 (1991) 497; K. Tsushima, T. Maruyama and A. Faessler, Nucl. Phys. A 537 (1992) 303.

[4] G. Chanfray and M. Ericson, Eur. Phys. J. A 25 (2005) 151.

[5] G. Chanfray, M. Ericson, nucl-th/0611042

[6] K. Saito, K. Tsushima and A. W. Thomas, Prog. Part. Nucl. Phys. 58 (2007) 1.

[7] C. Ratti, M. A. Thaler and W. Weise, Phys. Rev. D 73 (2006) 014019.

[8] R. Brockmann, W. Weise, Phys. Lett. B 367 (1996) 40.

[9] G.Q. Li,C.M. Ko, Phys. Lett. B 338 (1994) 118.

[10] B.D. Serot, J.D. Walecka, Adv. Nucl. Phys. 16 (1986) 1.

[11] B. ter Haar and R. Malfliet, Phys. Rep. 149 (1987) 207.

[12] R. Brockmann, R. Machleidt, Phys. Rev. C 42 (1990) 1965.

[13] T. Gross-Boelting, C. Fuchs, and Amand Faessler, Nucl. Phys. A 648 (1999) 105.

[14] R. Machleidt, K. Holinde, Ch. Elster, Phys. Rep. 149 (1987) 1.

[15] E. van Dalen, C. Fuchs, A. Faessler, Nucl. Phys. A 744 (2004) 227; Phys. Rev. Lett. 95 (2005) 022302; Phys. Rev. C 72 (2005) 065803.

[16] T. Klahn et al., Phys. Rev. C 74 (2006) 035802.

[17] D.R. Entem, R. Machleidt, Phys. Rev. C 66 (2002) 014002; Phys. Rev. C 68 (2003) 041001.

[18] D.R. Entem, R. Machleidt, Phys. Rev. C 68 (2003) 041001.

[19] E. Epelbaum, W. Glöckle, Ulf-G. Meissner, Nucl. Phys. A 747 (2005) 362.

[20] E. Epelbaum, Ulf-G. Meissner, W. Gloeckle, Nucl. Phys A 714 (2003) 535.

[21] S. R. Beane and M. J. Savage, Nucl. Phys. A 717 (2003) 91.

[22] E. Epelbaum, W. Glöckle, Ulf-G. Meissner, Eur. Phys. J. A 18 (2003) 499.

[23] E.G. Drukarev, E.M. Levin, Prog. Part. Nucl. Phys. 27 (1991) 77.

[24] P. Ring, Prog. Part. Nucl. Phys. 73 (1996) 193.

[25] O. Plohl, C. Fuchs, E. van Dalen, Phys. Rev. C 73 (2006) 014003.

[26] O. Plohl, C. Fuchs, Phys. Rev. C 74 (2006) 034325. 
[27] M. Birse, Phys. Rev. C 53 (1996) R2048.

[28] J.A. Tjon, S.J. Wallace, Phys. Rev. C 32 (1985) 267; Phys. Rev. C 32 (1985) 1667.

[29] C.J. Horowitz, B.D. Serot, Nucl. Phys. A 464 (1987) 613.

[30] F. Gross, J.W. van Orden, K. Holinde, Phys. Rev. C 45 (1992) 2094.

[31] F. Gross and A. Stadler, arXiv:0704.1229 [nucl-th].

[32] B. C. Lehnhart, J. Gegelia and S. Scherer, J. Phys. G 31 (2005) 89; [arXiv:hep-ph/0412092].

[33] R. Machleidt, Adv. Nucl. Phys. 19 (1989) 189.

[34] M. Procura, T. R. Hemmert, W. Weise Phys. Rev. D 69 (2004) 034505.

[35] G.E. Brown and M. Rho, Phys. Rep. 269 (1996) 333.

[36] C. Fuchs, H. Lenske, and H. H. Wolter, Phys. Rev. C 52 (1995) 3043.

[37] F. Hofmann, C. M. Keil, and H. Lenske, Phys. Rev. C 64 (2001) 034314.

[38] E. N. E. van Dalen, C. Fuchs and A. Faessler, Eur. Phys. J. A 31 (2007) 29.

[39] S. Typel and H. H. Wolter, Nucl. Phys. A 656 (1999) 331; T. Nikšić, D. Vretenar, P. Finelli, and P. Ring, Phys. Rev. C 66 (2002) 024306.

[40] T. Nikšić, D. Vretenar, P. Ring, Phys. Rev. C 66 (2002) 064302.

[41] A. Bulgac, G. A. Miller, M. Strikman, Phys. Rev. C 56 (1997) 3307.

[42] J. Gasser and H. Leutwyler, Phys. Rep. 87 (1982) 77.

[43] J. Gasser, H. Leutwyler and M. E. Sainio, Phys. Lett. B 253 (1991)252.

[44] C. Fuchs, Prog. Part. Nucl. Phys. 56 (2006) 1.

[45] J. Kuckei, F. Montani, H. Müther, A. Sedrakian, Nucl. Phys. A 723 (2003) 32.

[46] S.K. Bogner, A. Schwenk, R.J. Furnstahl, A. Nogga, Nucl. Phys. A 763 (2005) 59.

[47] SAID on-line program, R.A Arndt, R.L. Workman et al. see website http://gwdac.phys.gwu.edu/.

[48] E. Matsinos, hep-ph/9807395

[49] R. Koch, Nucl. Phys. A 448 (1986) 707.

[50] R. Thomas, T. Hilger and B. Kämpfer, arXiv:0704.3004 [hep-ph]; R. Thomas, S. Zschocke, B. Kämpfer, Phys. Rev. Lett. 95 (2005) 232301.

[51] B.L. Ioffe, Nucl. Phys. B 188 (1981) 317.

[52] P. Finelli, N. Kaiser, D. Vretenar, W. Weise, Nucl. Phys. A 735 (2004) 449. 